\title{
Calidad de vida relacionada con la salud: exclusión de la subjetividad
}

\author{
Health-related quality of life: Exclusion of subjectivity
}

Juan Pablo Lopera-Vásquez (https://orcid.org/0000-0001-6689-4834) ${ }^{1}$

${ }^{1}$ Unidad de

Neuropsicología, Instituto Neurológico de Colombia. Cl. 55 \#\#46-36. Medellín Antioquia Colômbia. juan.lopera@ neurologico.org.co

\begin{abstract}
Quality of life is a concept increasingly being used to assess the impact of chronic illnesses and the effectiveness of medical treatment options. The approach in the field of health is usually conducted using self-administered surveys, given the current high cost of instrumentalization of clinical practice. These instruments are useful for health professionals enabling them to analyze the effect of their interventions. Likewise, they are also relevant to public health, as they report on conditions of those with illnesses whose prevalence is increasing. Nevertheless, in this article, it is proposed that the quality of life approach is limited when it is carried out with structured instruments. It particularly reflects on how these instruments lack subjectivity, despite the fact that they are self-administered by patients. The limitations relating to quantification and transversality that arise when evaluating quality of life with these parameters are also analyzed. As an alternative to overcome these shortcomings, the narrative approach is proposed, as it makes it possible to report on changes experienced in subjectivity with the ongoing progress of the illness.
\end{abstract}

Key words Quality of life, Chronic illnesses, Narration
Resumo La Calidad de Vida es un concepto utilizado cada vez con más frecuencia para evaluar la afectación de las enfermedades crónicas y la efectividad de los tratamientos médicos. Su abordaje en el campo de la salud suele realizarse a través de cuestionarios autodiligenciados, dada la alta instrumentalización actual del ejercicio clínico. Esos instrumentos tienen utilidad para los profesionales de la salud, al permitirles analizar el efecto de sus intervenciones. Igualmente, son relevantes para la salud pública, en tanto informan acerca de las condiciones de quienes presentan enfermedades cuya prevalencia va en aumento. Sin embargo, en este artículo se plantea que el abordaje de la calidad de vida es limitado cuando se realiza con instrumentos estructurados. En particular se reflexiona acerca de cómo estos excluyen la subjetividad, pese a que son autodiligenciados por los pacientes. También se analizan las limitaciones concernientes a la cuantificación y a la transversalidad que se presenta al evaluar la calidad de vida con estas escalas. Como alternativa para superar estas falencias, se propone el abordaje narrativo, el cual permite dar cuenta de los cambios que se experimentan en la subjetividad con el transcurrir de la enfermedad.

Palabras clave Calidad de vida, Enfermedades crónicas, Narración 


\section{Introducción}

El número de personas con enfermedades crónicas y con discapacidad ha aumentado en las últimas décadas. En 1980 habían 108 millones de personas con diabetes y ahora la presentan 422 millones ${ }^{1}$. Igualmente creció la prevalencia de las enfermedades desmielinizantes ${ }^{2}$, demencias ${ }^{3} \mathrm{y}$ accidentes cerebrovasculares, condiciones que causan más discapacidad que letalidad ${ }^{4}$. El aumento de estas condiciones, y de otras que generan lesiones permanentes, incrementó las alteraciones funcionales, al punto que en el mundo hay más de mil millones de personas con discapacidad 5 .

En el manejo de esas enfermedades crónicas y discapacitantes es frecuente evaluar la Calidad de Vida Relacionada con la Salud (CVRS), la cual da cuenta de las dimensiones de la vida afectadas por enfermedades, accidentes, tratamientos o políticas de salud ${ }^{6}$. A nivel clínico su abordaje favorece el análisis de las áreas en que cada paciente presenta alteraciones relacionadas con su enfermedad y ayuda a evaluar el impacto de los tratamientos. Además, cuando no existe una cura definitiva, el mejoramiento de la CVRS es un objetivo terapéutico en sí mismo7.

Dada la importancia de la CVRS en el manejo de condiciones crónicas y discapacitantes, y el aumento en la prevalencia de estas, es esencial para la salud pública analizar las dificultades para abordar la CVRS. En ese análisis debe tenerse en cuenta que no hay consenso acerca de la definición de la CVRS y que esta no suele diferenciarse conceptualmente de la calidad de vida general ${ }^{8}$. Los pocos acuerdos existentes conciernen a su naturaleza multidimensional y subjetiva ${ }^{9}$, al aceptarse que la CVRS incluye varias dimensiones de la existencia y corresponde a lo informado por los pacientes.

$\mathrm{Al}$ indagar a estos acerca del efecto de las enfermedades y la discapacidad en su calidad de vida, se ha encontrado que discrepan con lo reportado por sus médicos y familiares ${ }^{10}$, y que su satisfacción con la vida no se correlaciona exactamente con la alteración en variables biomédicas ${ }^{7}$. Incluso se habla de la "paradoja de la discapacidad", consistente en la alta calidad de vida que reportan personas con enfermedades crónicas y severas limitaciones funcionales ${ }^{11}$. Esa discrepancia entre aspectos clínicos y lo informado por los pacientes reafirma que la CVRS debe abordarse desde la perspectiva de estos y no desde la del médico, en tanto desde su subjetividad construyen una valoración particular para su vida y sus problemas de salud.
En consecuencia, la calidad de vida suele evaluarse a través de autoreportes de los pacientes. Sin embargo, en este artículo se plantea que su subjetividad no logra expresarse en esas escalas, debido al carácter estructurado de esos instrumentos, a la cuantificación de la afectación y a su abordaje transversal. Estos tres aspectos se problematizan en este artículo, para luego proponer un abordaje narrativo, con el que se subsanarían las dificultades asociadas a los instrumentos estructurados.

\section{La evaluación por medio de instrumentos estructurados}

La evaluación de la CVRS se relaciona con la construcción de escalas, técnicas o instrumentos de medida ${ }^{12}$. Esto se asocia al ejercicio normalizador presente en las áreas médicas. En estas lo patológico suele determinarse a partir de la desviación respecto a valores de referencia poblacionales, para lo que se requiere de instrumentos válidos y consistentes que den cuenta de esa desviación ${ }^{13}$. Dado que para la CVRS no existen valores normales de referencia, esta suele evaluarse a través de instrumentos adecuados para comparar a sanos y enfermos. En consecuencia, el desarrollo de la CVRS derivó en la creación de instrumentos de medición, más que en clarificar el concepto ${ }^{14}$.

Esos instrumentos suelen ser genéricos o específicos $^{8}$. Los primeros se utilizan en personas con cualquier enfermedad y evalúan una concepción amplia de la salud, en la cual se indaga por el bienestar físico, emocional y social. Sin embargo, no hay un acuerdo acerca de lo que deberían incluir estas áreas ${ }^{15}$, ni respecto a la naturaleza y número de dimensiones que deben contener ${ }^{16}$. Debido a eso, algunas escalas también indagan acerca del bienestar con el entorno, la espiritualidad, la religión y las creencias personales'. En estos instrumentos se analiza el grado de bienestar o funcionalidad en cada dimensión, aunque en algunos también se calcula el bienestar resultante del conjunto de dimensiones ${ }^{17}$.

Por su parte, con los instrumentos específicos se evalúa el impacto de una condición de salud particular ${ }^{13}$. Con estos se indaga el malestar que provocan los síntomas o características de cada enfermedad. Debido a esta especificidad, son escalas aplicables solo en quienes presentan la patología o grupo de enfermedades de interés y no son utilizables en población general ${ }^{18}$. Pese a que con los instrumentos específicos no pueden realizarse comparaciones con la población sana, se tiene como referencia que la calidad de vida 
consiste en la ausencia de disfuncionalidad y de malestar. Es decir, el punto de normalidad con el que se compara es el de un estado de total bienestar en el que es posible realizar las actividades cotidianas.

Como puede apreciarse, estos instrumentos están muy ligados a las nociones de funcionalidad y de bienestar. En ellos se vislumbra una tendencia a considerar que la buena vida es contar con bienestar absoluto, ser funcional e independiente. Esa tendencia se basa en la perspectiva biomédica, en la que se considera que la salud corresponde al correcto funcionamiento orgánico y a la adecuada funcionalidad. Además, concuerda con la visión de la OMS, que define la salud como un estado de completo bienestar físico, mental y social ${ }^{19}$. Los instrumentos de CVRS se ciñen a esa definición, pues cuando estos muestran que se conserva el bienestar y la funcionalidad, se concluye que se posee buena calidad de vida. Desde esta perspectiva la buena vida es contar con buena salud. Cuando se tienen enfermedades, una buena vida es aquella en la que estas no afectan el bienestar y la funcionalidad. Debido a esto, se cuestiona si los instrumentos para evaluar CVRS en efecto abordan la calidad de vida o si son medidas generales de salud ${ }^{20}$.

Igualmente, se cuestiona el énfasis de la CVRS en el funcionalismo y la falta de marcos teóricos que expliquen la compartimentación en dimensiones que conforman la salud ${ }^{21}$. En medio de esos cuestionamientos, a los instrumentos para evaluar la CVRS se les sigue exigiendo dar cuenta de la funcionalidad y el bienestar ${ }^{22}$. Estos se construyen a partir del conocimiento clínico acerca de las enfermedades, de sus síntomas y problemáticas asociadas. Para construirlos se estudian diversos grupos poblacionales, con el fin de garantizar que sean válidos para toda la población, en el caso de los genéricos, o para el segmento poblacional con enfermedades particulares, en el caso de los específicos. En su construcción también se utilizan procedimientos cualitativos para determinar áreas y preguntas a incluir, así como para definir el número de ítems que permiten abordar lo que es importante para cada persona ${ }^{23}$.

Esos esfuerzos por construir escalas válidas no son suficientes para incluir la subjetividad de quienes las responden. Una de las razones es que suele asumirse que todas las dimensiones en el instrumento tienen una importancia similar para cada persona ${ }^{24}$. Otra razón es la reducción de la noción de calidad de vida a ser funcional, tener bienestar y carecer de enfermedades. Todo aquel que no otorgue gran importancia a estos aspectos, encontrará poco adecuados esos instrumentos para explicar el efecto de la enfermedad en su vida, tal como debe sucederle a quienes encuentran beneficios en su condición de salud. En consecuencia, las escalas de CVRS son siempre incompletas para dar cuenta de la subjetividad, en tanto en esta se expresa lo singular de cada persona. Sin embargo, esos instrumentos subestiman lo singular, pues en ellos poco se tiene en cuenta que cada individuo otorga un significado particular a sus síntomas y disfuncionalidades, de acuerdo a lo que representan para su vida.

Para comprender la omisión de la subjetividad, analicemos el caso de las escalas para personas con trastornos del movimiento. En estas se pregunta si los síntomas motores afectan la realización de actividades como trabajar, desplazarse o hacer ejercicio. Responder afirmativamente deriva en una baja puntuación en la CVRS. Pero quien así responde podría no sentirse muy afectado, en tanto no hacía deporte, su trabajo le molestaba o no le interesa moverse a lugares distantes. Es posible que esa persona conteste que se siente bien, pese a su enfermedad. Su cuestionario de CVRS mostrará entonces que unas dimensiones se afectan por limitaciones motoras y que no están afectadas otras dimensiones relacionadas con la satisfacción general. Si por el contrario, esa persona responde que su vida no es satisfactoria, así eso no se relacione con su enfermedad, el resultado del cuestionario mostrará que se encuentra afectada su CVRS. Ese resultado estará atravesado por el supuesto de que eso se debe a su enfermedad, aunque el instrumento no logra dar cuenta de los motivos reales.

Aspectos importantes para la subjetividad, como son los motivos, razonamientos, significados y valoraciones singulares de cada individuo, no logran abordarse con estos cuestionarios. Es paradójico que la CVRS sea subjetiva, pero que los instrumentos para evaluarla no abarquen estos asuntos de la subjetividad. Eso puede deberse a que la evaluación de la calidad de vida exige metodologías en las que no se parta de presupuestos generalizados, como son aquellos en los que al bienestar, a la funcionalidad y a la ausencia de enfermedad se les otorga un lugar fundamental para todas las personas. Además, se requiere metodologías en las que cada individuo pueda definir las áreas importantes para sí, y en las que no deba cuantificar el bienestar experimentado, pues la cuantificación también limita el abordaje de la CVRS. 


\section{Limitaciones debidas a la cuantificación, la validez y la confiabilidad}

Los indicadores para medir la CVRS son notablemente bioestadísticos, psicométricos y económicos, fundamentados en una lógica de costo-beneficio ${ }^{25}$. El apelar a estos se asocia a la necesidad de cuantificación, tan común en todos los ámbitos en que se toman decisiones con bases científicas. A muchos les tranquiliza tener información en términos numéricos, pues pareciera que al cuantificarse un fenómeno se obtuviera mayor certeza y conocimiento. Sin embargo, eso no sucede con la CVRS, pues su cuantificación no ofrece suficiente información y podría ser un obstáculo para vislumbrar asuntos más relevantes. De hecho, algunos autores sugieren no buscar un resultado global para la calidad de vida, en tanto a esta la conforman dimensiones variadas, ni que es práctico evaluar todas las dimensiones ${ }^{6}$.

Ante ese cuestionamiento se ha propuesto que la cuantificación sea solo para cada dimensión. Sin embargo, dentro de estas también hay particularidades muy variadas. Incluso, hay casos en los que la enfermedad afecta el bienestar a la vez que lo aumenta. Esa situación se presenta cuando la patología conlleva beneficios y aprendizajes. Ese tipo de situaciones no se incluyen en los instrumentos tradicionales, pues suele considerarse que una enfermedad solo resta bienestar, no que lo aumenta. Además, esos aprendizajes y ganancias no obedecen estrictamente a un bienestar y no logran cuantificarse con facilidad. De hecho, muchos asuntos de la vida no logran ponderarse, pues no es fácil cuantificar qué tanto afecta el que una enfermedad nos impida trabajar, nos haya dejado ciegos o sin una extremidad. Quizás, acerca de esos asuntos no es un número lo que mejor orienta, sino una explicación o una narración acerca del impacto de esa situación para la propia vida.

A este planteamiento podría objetársele que no suelen ser los pacientes quienes cuantifican su bienestar o funcionalidad. En efecto, muchas preguntas en estas escalas exigen respuestas dicotómicas, en las que debe responderse si la enfermedad afecta o no lo hace. También se utilizan esquemas en los que debe ubicarse un punto específico dentro de una línea que va desde la peor vida posible hasta la mayor calidad de vida. Las preguntas más frecuentes tienen formato Likert, en las que se escoge si el síntoma limita mucho, poco o nada; o si la molestia nunca se presenta, lo hace en ocasiones, con frecuencia o siempre está presente. Esas opciones de respuesta, sean dico- tómicas, con formato Likert o derivadas de un esquema visual, se traducen a un número dentro de un continuum que va de cero a cien, en donde el valor mínimo corresponde a la mayor afectación y el valor máximo a la inexistencia de alteración.

El extrapolar las respuestas a valores cuantitativos genera dudas acerca de la representatividad del número obtenido. Este puede ser un indicador erróneo de la respuesta otorgada por alguien que considere que es muy molesto aquello que solo le afecta en ocasiones. Si al indagarse por ese asunto, las posibilidades de respuesta del instrumento son: "nunca, en ocasiones, con frecuencia, casi siempre y siempre", esa persona posiblemente responda que eso le afecta solo en ocasiones. Esa respuesta indica baja afectación para la CVRS, pese a lo mucho que podrían incomodar esas pocas ocasiones en que se presenta la problemática indagada. Incluso, es posible que su respuesta fuese diferente al valor obtenido, si el instrumento solicitara que el paciente cuantificara directamente ese ítem.

Este tipo de dificultades no parece interesar a quienes desarrollan estas escalas, ni a quienes las utilizan, en tanto consideran que esos instrumentos revelan aspectos de la subjetividad de quienes los diligencian. En efecto, podría asumirse que al autodiligenciar un cuestionario se da cuenta de la subjetividad, por el mero hecho de responder los interrogantes. Sin embargo, asumir eso sería ignorar que la cuantificación del bienestar y de la funcionalidad excluye a todo aquello de la subjetividad que no logra abordarse en las preguntas y opciones de respuesta que brindan las escalas.

Eso que queda excluido es lo que molesta al diligenciar cuestionarios acerca de aspectos en los que la subjetividad es relevante, pues con frecuencia no se logran encuadrar las experiencias propias dentro de las opciones de respuesta. Para lograr ese encuadre se requieren más preguntas y diferentes opciones de respuesta, que amplíen las posibilidades para mencionar otros asuntos. A esta inquietud podría objetársele que en los instrumentos que cumplen criterios de validez, las preguntas incluidas serían suficientes. Ante esa objeción cabe plantear que la validez es un concepto con una lógica contraria a la de la subjetividad. Si esta última habla de lo singular, la primera se refiere a lo que es aplicable a todos. Los instrumentos de CVRS se consideran válidos al ser representativos de ese constructo teórico, el cual se plantea como un concepto válido para todos. Por tanto, las dimensiones en ellos serían las que en general importan a toda la población, pese a que lo valioso para cada ser humano es 
subjetivo. Es decir, lo valioso para cada uno es singular y quizás poco valioso para los demás. En consecuencia, cabe preguntarse si es posible que realmente existan cuestionarios válidos de CVRS.

Además de la validez, a estos instrumentos también puede cuestionársele su confiabilidad. $\mathrm{Al}$ respecto, suele considerarse que extrapolar las respuestas a un valor cuantitativo brinda confiabilidad, en tanto es difícil para las personas cuantificar la afectación que les genera un síntoma particular. Por eso, se asume que las opciones de respuesta en las escalas Likert, facilitan su diligenciamiento y aumentan la confiabilidad. Sin embargo, esas opciones no son suficientes para obtener una apreciación subjetiva más adecuada, pues tampoco es fácil responder si un síntoma o particularidad de una enfermedad afecta mucho, poco o nada en ciertos aspectos de la vida, en tanto eso depende también de asuntos contextuales a cada persona. Por ejemplo, una limitación motriz afecta más si no se tiene quién colabore o si hay barreras adicionales que aumentan la discapacidad. Igualmente, habrá quienes consideren que sus limitaciones motrices afectan solo cuando hay decaimiento anímico o cuando hay otras personas que juzgan sus intentos de realizar sus actividades.

El contexto es fundamental para determinar si algo afecta mucho o poco, pues influye en la subjetividad. Ese contexto incluye asuntos del entorno físico, social y personal de cada individuo, lo que se siente y piensa en un momento dado, lo que sucede en ese instante y las interacciones con los demás. Incluso, el momento histórico y la cultura aportan a la subjetividad ${ }^{26}$. Esos factores emergen en esta como expresión del ser, para dar cuenta de un sujeto en continua transformación. La subjetividad se expresa al responder los instrumentos de CVRS, sin que estos le permitan desplegar lo que la conforma, debido a que suelen ser instrumentos con buena confiabilidad test-retest e interevaluador. Eso implica que las respuestas de un sujeto particular no deben variar ante diferentes evaluadores ni en momentos diferentes, entre los cuales su salud no ha cambiado.

La confiabilidad, al igual que la validez, sigue una lógica contraria a la de la subjetividad. Un instrumento confiable garantiza conocer características propias y constantes de quien lo diligencia. Pero la subjetividad, además de incluir lo que es constante, incluye lo que es cambiante del sujeto. Sin embargo, eso que cambia es lo que elimina la confiabilidad en estos instrumentos. La confiabilidad se le exige a los instrumentos estructurados, pese a que al obtenerla se ignora la movilidad de la subjetividad. A esto se añade que la confiabilidad puede incluso dificultar que se expresen esas características sólidas o permanentes del sujeto, lo cual es contrario a lo que ella misma busca. Eso se debe a que la confiablidad puede desprenderse de lo limitantes que son las opciones de respuesta, lo cual deriva en que quienes diligencian las escalas respondan siempre de la misma manera, sin permitir que se expresen características móviles de su subjetividad, ni otros asuntos esenciales de sí. El que alguien conteste siempre igual, cada que diligencia el instrumento, no quiere decir que se identifique con la opción escogida. Esa persona podría sentir que no tuvo la opción de respuesta más adecuada y que la prueba no abordó lo importante.

Lo que es fundamental no logra expresarse en una respuesta a una pregunta puntual. Una condición crónica o discapacitante puede conllevar enormes dosis de sufrimiento y de alteración en la cotidianidad para un sujeto en particular. Sin embargo, otra persona con la misma condición, podría contestar que no se ha alterado su vida lo suficiente, pese a las grandes dificultades que conlleva la enfermedad. Las respuestas de esta última persona podrían ser siempre las mismas, aunque estas no reflejen realmente esa afectación. El hecho de que responda de la misma manera indica que el instrumento es confiable. Pero esa respuesta, en la que atenúa la afectación, podría deberse a que considere que esa es la voluntad de Dios y que debe aceptarla. Su creencia religiosa puede ser una característica inamovible de sí, que atraviesa sus respuestas, generándole la sensación de que su situación no es tan negativa, pese a que presente dolores, disfuncionalidades y síntomas tormentosos. Igual situación puede hipotetizarse acerca de otras creencias y razonamientos, que podrían modificar la forma de responder los cuestionarios, derivando en conclusiones inadecuadas acerca de su CVRS.

La cuantificación puede ser útil para abordar otros asuntos humanos, en los que se mide en una sola dimensión, como sucede con la edad, el peso o la estatura. Pero el abordaje de la subjetividad no está relacionado con dimensiones precisas ni cuantificables. Los múltiples asuntos que la conforman y la atraviesan, así como su movilidad, la hacen inadecuada para abordarla con instrumentos estructurados y le restan precisión a cualquier intento de medir lo que la constituye. Si la CVRS es subjetiva, entonces deben tenerse presente estas características de la subjetividad. Además, debe cuestionarse qué tan adecuado es 
confiar en la cuantificación de un concepto que incluye asuntos con cualidades poco concretas, tangibles y ponderables. Abordar la subjetividad y la calidad de vida implica moverse en el ámbito de lo inexacto e incierto. En consecuencia, las seguridades que brinda la cuantificación de la CVRS no son tan sólidas, pues los números pueden reflejar un abordaje incompleto e impreciso con el que se constriñen las respuestas de las personas. Ese abordaje no refleja rigor académico, así esos números provengan del uso de instrumentos válidos y confiables. A estas falencias, acerca de la evaluación y cuantificación de la CVRS, se asocia la transversalidad del abordaje, que implica otras limitantes.

\section{El abordaje transversal}

En los cuestionarios específicos para evaluar la CVRS, las preguntas aluden al impacto de la enfermedad durante la última semana o el último mes. Incluso, suelen contener preguntas acerca de la percepción del cambio durante el último año. Estas escalas no incluyen un análisis longitudinal, en tanto solo indagan por qué tanto afecta la patología en ese tiempo específico. Sin embargo, la cronicidad de las enfermedades y de la discapacidad conlleva que con estas pueda vivirse durante años, atravesándose etapas diferentes en las que se dan transformaciones relacionadas con la condición de salud y el devenir existencial.

Quienes presentan enfermedades crónicas y discapacitantes atraviesan momentos que podrían incluir fases sintomáticas y con desconocimiento de las razones de los síntomas. Luego aparece el diagnóstico, el cual puede frustrar, al encontrarse con una patología incapacitante y sin cura. Pero también puede tranquilizar, cuando este se presenta luego de un largo período de síntomas cargado de incertidumbre. Con el paso del tiempo pueden aparecer etapas de mejoría o empeoramiento, según la patología o el evento presentado. Cada persona experimenta esas fases de forma diferente, y cada una afectará de manera particular, de acuerdo a factores como la personalidad del paciente, su resiliencia, edad, planes para su vida, red de cuidadores, actividades cotidianas, entre otros asuntos particulares a cada caso.

Estos asuntos tienen importancia variable según el momento histórico que se atraviesa. Por eso, hay personas para quienes enfrentarse a la incapacidad laboral es una tragedia, de acuerdo a la importancia del trabajo en sus vidas, ya que lo disfrutan, reciben reconocimiento con este o tienen muchas obligaciones económicas. Sin embar- go, esa importancia puede variar, de acuerdo a la aparición de otras actividades en la cotidianidad, de las resignificaciones que se hace de la vida y de la transformación en las relaciones sociales y consigo mismo. Estos cambios relacionados con el mundo laboral son ignorados en los cuestionarios sobre CVRS, en los que al respecto, se indaga solo por qué tanto la condición de salud afecta para trabajar. Quien responde esa pregunta probablemente lo hará analizando su funcionalidad. Pero la pregunta no indaga por todo lo que gira en torno al trabajo y que influye en la afectación que se busca evaluar.

Para comprender cómo se afecta la dimensión laboral es necesario preguntar por otros asuntos contextuales y temporales. El obtener una respuesta acerca del impacto en la capacidad para trabajar, no es suficiente para comprender lo que sucede en ese aspecto de la vida. Esa respuesta puntual funciona como una fotografía de un detalle, a la que se le recortó su contorno. Esa imagen da un sentido diferente al que se obtiene cuando se le ubica en el entorno que la envuelve. Igual situación se presenta con esas respuestas que no se ubican en el contexto que brinda una historia de vida. El sentido que se adquiere con las respuestas a instrumentos en los que se abordaje transversalmente, es muy diferente al sentido que tomarían si se les ubicara dentro de un contexto amplio, que no se capta con abordajes transversales, sino con una mirada longitudinal. Eso mismo sucede con las preguntas que se hacen acerca de otras dimensiones diferentes a la laboral.

Los abordajes clínicos en los que se indaga por el estado actual de salud y por el efecto que las enfermedades y la discapacidad generan en ese momento, son limitados si no se relacionan con las expectativas y las experiencias de las personas. Precisamente, se ha planteado que la interacción entre estas debe ser analizada, para así evitar que la CVRS sea solo una medición de la salud general $^{27}$. Pero no son únicamente las expectativas y las experiencias las que deben abordarse, sino todo el contexto que las rodea y que se aprecia en la historia de vida. Sin embargo, los instrumentos de CVRS, tan utilizados porque permiten abordar rápidamente asuntos amplios, pueden llevarnos a cortar los lazos con los contextos, acontecimientos, motivos y situaciones, que brindan información pertinente para comprender los asuntos indagados.

Esta reflexión acerca de cómo la transversalidad corta lazos con la historia de vida, muestra lo inadecuado del instrumentalismo que envuelve a la evaluación de la CVRS, ya que para indagar 
acerca de algunos asuntos son poco útiles los instrumentos estructurados. Para abordar temáticas de la vida privada, como son la sexualidad, las frustraciones, fracasos, idealizaciones, entre otras, se requiere de métodos que muestren los cambios a lo largo del tiempo, a la vez que generen confianza y provoquen apertura al tema. En los instrumentos estructurados esa apertura falla y lleva a que las personas escojan alguna de las opciones de respuesta en el instrumento, en tanto eso es lo que se les solicita. Sin embargo, sus respuestas quizás reflejen poco de lo que sucede en sus vidas. Por el contrario, puede que un pequeño relato, en el que se da cuenta de esas situaciones, provea más información que todas las respuestas obtenidas con la escala de CVRS.

\section{Conclusión: Incorporar metodologías narrativas}

Las escalas para evaluar la CVRS se utilizan debido a la confianza actual en los instrumentos estructurados y en la obtención de valores precisos y numéricos. Sin embargo, los médicos dudan acerca de la efectividad de los datos obtenidos al evaluar la calidad de vida $^{28}$ y cuestionan la validez e importancia de esas medidas ${ }^{29}$. Ese escepticismo puede deberse a la forma en que se aborda la CVRS, la cual, de acuerdo a las reflexiones en este artículo, deriva en un conocimiento impreciso, en el que la subjetividad no tiene lugar.

Pese a las limitaciones mencionadas, cuando los médicos desean evaluarla, optan por alguna de las escalas existentes. Eso puede deberse a que son de fácil aplicación, pues los pacientes las diligencian. No es casualidad que los instrumentos más comunes son los más abreviados, pues el tiempo de aplicación influye en su utilización en la práctica clínica ${ }^{30}$. Precisamente, lo limitado del tiempo de consulta es uno de los motivos de los médicos para no incorporar instrumentos de CVRS en su práctica clínica ${ }^{28}$. Cuando los utilizan prefieren las escalas breves, que permiten en pocos minutos abordar muchas áreas. Eso puede deberse a que con esos instrumentos puede explorarse un concepto multidimensional, cuyo abordaje con otras metodologías tardaría mucho tiempo.

El evaluar la CVRS con instrumentos estructurados conlleva el riesgo de que a los pacientes se les deja de ver como seres complejos inaprehensibles en su totalidad, y se les considere como el mero conjunto de dimensiones predefinidas en el instrumento. Esa forma de abordaje olvida que la calidad de vida es lo que cada individuo determina que es ${ }^{24}$, lo cual incluye las dimensiones que cada uno considere importantes, y no las que predefine el instrumento.

El abordar la calidad de vida originalmente fue una forma de humanizar la atención, al otorgar la palabra al enfermo para que exprese sus perspectivas acerca del impacto de la enfermedad y del tratamiento. Sin embargo, al instrumentalizarse el ejercicio clínico, la subjetividad volvió a excluirse, tomando importancia el resultado numérico y el área en que la enfermedad es más agresiva, o en la que los tratamientos han fallado. En la actualidad parece no importar que el instrumento excluya la subjetividad. Lo que importan son los números, que otorgan cientificidad y rigor a la exploración clínica. Mientras que en el ámbito clínico se valoren tanto los instrumentos estructurados y la cuantificación, estas escalas seguirán vigentes.

Contrario a esa situación, la historia de la práctica clínica muestra que al consultar, los pacientes expresan sus vivencias y sensaciones sin necesidad de instrumentos estructurados. Lo hacen en medio de la conversación espontánea derivada del interés que perciban por parte de los médicos hacia sus problemáticas. Pero cuando el encuentro clínico se instrumentaliza, se pierde la fluidez de la conversación. La instrumentalización lleva a que el médico se dedique a examinar y a recolectar datos relevantes para su ejercicio clínico. Por su parte, quien consulta se dedica a responder lo que se le pregunta. Eso sucede con las escalas de evaluación de CVRS. El médico las entrega a sus pacientes, les explica cómo diligenciarlas y estos acatan ese pedido. En el mejor de los casos, el médico hace las preguntas del instrumento y el paciente responde, pero aun así esa instrumentalización de la relación clínica entorpece el diálogo fluido.

Si se busca superar esas limitaciones, debe optarse por otras formas de abordaje. Una posibilidad para estudiar la CVRS, sin limitar la subjetividad, es utilizar metodologías cualitativas. En estas la validez consiste en la habilidad para contar o relatar ${ }^{31}$. En vez de apelar a instrumentos estructurados, el médico podría generar condiciones para relatar libremente. Eso se logra a través de conversaciones abiertas acerca de las vivencias con la enfermedad o la discapacidad, en las que el profesional motiva a los pacientes a contar sus experiencias. Al relatar, estos crean narraciones en las que son protagonistas. Las narraciones son especialmente útiles para estudiar las experiencias, las relaciones y la singularidad 
de cada acción, así como las motivaciones, deseos y propósitos ${ }^{32}$, en tanto ellas tienen una trama que articula las vivencias. En las narraciones los pacientes expresan acciones en relación a su condición de salud, así como lo que motivó esas acciones y las circunstancias que rodearon a la vivencia. Igualmente, pueden expresar las acciones de otras personas y su efecto.

Esos relatos pueden girar en torno a su condición de salud o a otros asuntos. Con ellos el médico puede comprender mejor lo que les acontece a sus pacientes y entender cómo los síntomas y disfuncionalidades se articulan con diferentes dimensiones existenciales. Las narraciones permiten dar sentido a los efectos que pueda tener el déficit en el funcionamiento del organismo. Así, cuando alguien consulta al médico, este puede comprender mejor la afectación de la CVRS, debida a síntomas, disfuncionalidades y severidad de la enfermedad, y la afectación debida a otros asuntos, como son el no recibir apoyo de los cuidadores, la presencia de barreras arquitectónicas o sociales, o la negación de tratamientos por parte del sistema de salud. Para saber acerca de estos asuntos se requiere conocer la historia de vida del paciente, así como la evolución de su condición $y$ de las formas de vincularse con su enfermedad, sus cuidadores, sus actividades y con los servicios asistenciales.

Para saber acerca de esos asuntos hay que asumir un abordaje que permita dar cuenta de lo que ocurre en el tiempo y de la temporalidad de la existencia. Es decir, de la cronología de los eventos asociados a la enfermedad, así como de la forma en que se evocan esos eventos, de lo que se atiende de estos en el presente y de lo que se espera para el futuro ${ }^{33}$. La temporalidad de cada persona es diferente, pues los recuerdos y expectativas son singulares, así como los motivos por los que les afecta una patología. Es necesario entonces que en los ejercicios clínicos adoptemos metodologías que den cuenta de estos asuntos, como lo hacen los abordajes narrativos. En conclusión, en el ejercicio clínico debemos retomar el diálogo espontáneo para abordar la CVRS. Eso podría implicar el abandono de los instrumentos actuales, que son artificios que supuestamente permiten conocer a quien consulta. Artificios que derivan en un conocimiento muy artificial.

Pese a la artificialidad y falta de representatividad que pueden conllevar los números, los instrumentos cuantitativos han permitido dar cuenta de rasgos generales presentes en cada patología. Por tanto, abandonar el abordaje cuantitativo puede derivar en que no se vislumbren generalidades importantes. Un buen ejercicio clínico es aquel en el que el profesional se mueve con sabiduría entre las generalidades reportadas para cada patología y las particularidades de cada paciente. Además, implica tener presente la subjetividad, evitando excluir la psique del paciente, por focalizarse solo en lo que muestra su conducta; e implica evitar la exclusión del sujeto, por focalizar solo su enfermedad.

La evaluación de la CVRS en el ejercicio clínico exige entonces ser capaz de articular instrumentos que cuantifiquen y que cualifiquen lo que le sucede a cada paciente. Eso exige del clínico capacidad para apelar a instrumentos cuantitativos y cualitativos. El apoyarse en ambos, logrando interpretar la información que le brindan, es lo que le permitirá conocer mejor a sus pacientes y tomar buenas decisiones de tratamiento junto a estos. En consecuencia, el clínico debe tener presente que no es posible abordar la CVRS solo cuantitativamente. La subjetividad desde la cual contesta cada persona es tan compleja, que incluso incorporando metodologías narrativas tampoco se logre dar de esta, en tanto es móvil e imposible de aprehender en su totalidad. 


\section{Agradecimientos}

Al Departamento Administrativo de Ciencia, Tecnología e Innovación (Colciencias), que patrocinó la formación doctoral del autor y la realización de la investigación Reconocimiento como Potenciador de la Autonomía y la Calidad de Vida. Relato de un viaje alrededor de la esclerosis múltiple, de la cual se desprende este artículo. Igualmente, al Instituto Neurológico de Colombia, que apoyó su realización y el abordaje de los participantes.

\section{Referencias}

1. Organización Mundial de la Salud (OMS). Informe mundial sobre la diabetes. Geneva: OMS; 2016.

2. Browne P, Chandraratna D, Angood C, Tremlett H, Baker C, Taylor BV, Thompson AJ. Atlas of Multiple Sclerosis 2013: A growing global problem with widespread inequity. Neurology 2014; 83(11):1022-1024.

3. Annear MJ, Toye C, McInerney F, Eccleston C, Tranter B, Elliott K-E, Robinson A. What should we know about dementia in the 21st Century? A Delphi consensus study. BMC Geriatr [Internet]. 2015 [citado el 3 de abril de 2017]; 15. Disponible en: http://www. ncbi.nlm.nih.gov/pmc/articles/PMC4326452/

4. Flick C. Stroke rehabilitation. Stroke outcome and psychosocial consequences. Arch Phys Med Rehabil 1999; 80(Supl. 1):S21-26.

5. Organización Mundial de la Salud (OMS), Banco Mundial (BM). Informe mundial sobre la discapacidad [Internet]. 2011 [citado el 4 de abril de 2017]. Disponible en: http://www.who.int/disabilities/world_report/2011/es/

6. Fayers P, Machin D. Quality of Life. Assessment, Analy sis and Interpretation. Chichester: John Wiley \& Sons; 2000.

7. Alonso J. La Medida de la Calidad de Vida Relacionada con la Salud en la Investigación y la Práctica Clínica. Gac Sanit 2000; 14(2):163-167.

8. Urzúa A. Calidad de vida relacionada con la salud: Elementos conceptuales. Rev Médica Chile 2010; 138(3):358-365.

9. WHOQOL Group. The World Health Organization Quality of Life Assessment (WHOQOL): Position Paper from the World Health Organisation. Soc Sci Med 1995; 41(10):1403-1409.

10. Pearlman R, Uhlmann R. Quality of life in chronic diseases: perceptions of elderly patients. J Gerontol 1988; 43(Supl.):M25-30.

11. Albrecht G, Devlieger P. The Disability Paradox: High Quality of Life against All Odds. Soc Sci Med 1999; 48(8):977-988.

12. Fernández-Mayoralas G, Rojo F. Calidad de vida y salud: planteamientos conceptuales y métodos de investigación. Territoris 2005; 5:117-135.

13. Velarde-Jurado E, Avila-Figueroa C. Consideraciones metodológicas para evaluar la calidad de vida. Salud Pública de México 2002; 44(5):448-463.

14. Bullinger M. Cognitive Theories and Individual Quality of Life Assessment. En: Joyce C, McGee H, OBoyle C, editores. Individudal Quality of Life Approaches to Conceptualisation and Assessment. Amsterdam: Harwood Academic Publishers; 1999. p. 29-40.

15. Michalos A. Social Indicators Research and HealthRelated Quality of Life Research. Soc Indic Res 2004; 1:27-72.

16. Rapley M. Quality of Life Research. A Critical Introduction. London: SAGE Publications; 2003.

17. Cummins RA. The Comprehensive Quality of Life Scale - Adult: Manual. Melbourne: School of Psychology, Deakin University; 1997.

18. Tudela LL. La calidad de vida relacionada con la salud. Aten Primaria 2009; 41(7):411-416. 
19. Organización Mundial de la Salud (OMS). Constitución de la Organización Mundial de la Salud [Internet]. 1948. [citado el 3 de abril de 2017]. Disponible en: http:// www.who.int/governance/eb/who_constitution_sp.pdf

20. Carr AJ, Higginson I. Measuring quality of life Are quality of life measures patient centred? BMJ 2001; 322(2):1357-1360.

21. Leplège $A$, Hunt $S$. The problem of quality of life in medicine. JAMA 1997; 278(1):47-50.

22. Fernández-López JA, Hernández-Mejía R, Siegrist J. El perfil de calidad de vida para enfermos crónicos (PECVEC): un método para evaluar bienestar y funcionalismo en la práctica clínica. Aten Primaria 2001; 28(10):680-689.

23. Alarcon AM, Muñoz S. Medición en salud: Algunas consideraciones metodológicas. Rev Médica Chile 2008; 136(1):125-130.

24. Hickey A, O’Boyle C, McGee H, Joyce C. The Schedule for the Evaluation of Individual Quality of Life. En: Joyce C, O’Boyle C, McGee H, editores. Individual Quality of Life: Approaches to Conceptualisation and Assessment. Amsterdam: Harwood Academic Publishers; 1999. p. 119-134.

25. Minayo MCS, Hartz ZMA, Buss PM. Qualidade de vida e saúde: um debate necessário. Cien Saude Colet 2000; 5(1):7-18.

26. Vargas R. Sujeito, subjetividade e modos de subjetivação na contemporaneidade. Rev Psicol UNESP 2009; 8(2):110-117.

27. Carr AJ, Gibson B, Robinson P. Is quality of life determined by expectations or experience? BMJ 2001; 322(7296):1240-1243.
28. Skevington SM, Day R, Chisholm A, Trueman P. How much do doctors use quality of life information in primary care? Testing the Trans-Theoretical Model of behaviour change. Qual Life Res 2005; 14(4):911-922.

29. Tudela LL, Ferrer AR, Martínez MR, García JC. Evaluación de la calidad de vida relacionada. Med Clin Barc 2000; 114(Supl. 3):76-80.

30. Patrick D, Deyo R. Generic and disease specific measures in assessing health status and quality of life. Med Care 1989; 27(3 Supl.):217-232.

31. Kirk J, Miller ML. Reliability and Validity in Qualitative Research. Beverly Hills: SAGE Publications; 1986.

32. Bolívar A, Domingo J. La investigación biográfica y narrativa en Iberoamérica: Campos de desarrollo y estado actual. Forum Qual Soc Res 2006; 7(4). Disponible en: http://www.qualitativeresearch.net/index. $\mathrm{php} / \mathrm{fqs} /$ article/view/161/357

33. Ricoeur P. Historia y narratividad. Barcelona: Ediciones Paidós Ibérica; 1999.

Artigo apresentado em 02/07/2017

Aprovado em 22/05/2018

Versão final apresentada em 24/05/2018 